\title{
Deep Brain Stimulation of the Periaqueductal Gray for Treatment of Nociceptive Pain: Tryptophan Loading and the Serotonergic Circuit of Pain
}

\author{
Yashar Kalani ${ }^{1}$, Maziyar A. Kalani ${ }^{2}$, Eric E. Sabelman ${ }^{3}$, Damien Carracedo, Gary Heit ${ }^{5}$ \\ 1. Division of Neurological Surgery, Barrow Neurological Institute 2. Department of Neurosurgery, \\ Stanford University School of Medicine 3. Department of Neurosurgery, The Permanente Medical Group, \\ Redwood City, CA 4. 5. The Permanente Medical Group
}

$\square$ Corresponding author: Yashar Kalani, stemcelldoctor@gmail.com

Disclosures can be found in Additional Information at the end of the article

\section{Abstract}

Background: Pain and pain-related diseases pose a major treatment challenge. Current medical treatments frequently provide inadequate pain control to patients, or cause dependence and other unwanted side-effects. Despite initial failures of open-label trials to show efficacy in the use of deep brain stimulation (DBS) for pain, several groups have reported utility of this modality on carefully selected patients. There are several characterized pain pathways in operation in the human brain. A common theme to the pathways thus far identified is their dependence on the neurotransmitter serotonin for modulation of pain. Deep brain stimulation for pain may well activate one of the serotonin-dependent pathways to stimulate relief. Greater experience, and improvements in both technology and patient selection, has paved the way for the potential use of electrical stimulation of subcortical structures to manage intractable and nociceptive pain syndromes and also for the mechanistic study of the pathways involved in the symptomatic relief of pain caused by stimulation.

Methods: We preoperatively loaded four patients with 4 grams of L-tryptophan, and implanted DBS electrodes in the periaqueductal gray (PAG).

Results: Upon loading the patients with 4 grams of L-tryptophan two weeks preoperatively and the continual use of tryptophan at doses greater than 2.5 grams, we note a significant improvement in the pain symptoms of all of our patients implanted at the PAG. Upon decrease in L-tryptophan dose below 2.5 grams, every patient experienced return of their pain symptoms despite PAG stimulation. The average length of followup is 9.75 months with a range of three to 14 .

Conclusions: Tryptophan loading of the patient prior to PAG electrode placement, and the continued use of tryptophan postoperatively correlates with better outcomes and more significant pain reduction. Tryptophan probably increases serotonin levels and results in activation of anti-nociceptive pain pathways at the PAG.

Kalani et al. This is an open access article distributed under the terms of the Creative Commons Attribution License CC-BY 3.0., which permits unrestricted use, distribution, and reproduction in any medium, provided the original author and source are credited.
Categories: Pain Management, Neurosurgery

Keywords: serotonin, pain, deep brain stimulation, periaqueductal gray, tryptophan

\section{Introduction}

How to cite this article

Kalani Y, Kalani M A, Sabelman E E, et al. (January 09, 2010) Deep Brain Stimulation of the

Periaqueductal Gray for Treatment of Nociceptive Pain: Tryptophan Loading and the Serotonergic Circuit of Pain. Cureus 2(1): e7. DOI 10.7759/cureus.7 
In the United States alone, an estimated 50 million people suffer from chronic pain. Although some pain is responsive to medication, other types of pain are refractory to most common modalities available. Recent advances in deep brain stimulation technology have paved the way for the use of electrical stimulation of cerebral and subcortical nuclei to manage intractable and nociceptive pain syndromes.

The use of electrical stimulation for the treatment of pain has a history of nearly half a century, with the introduction of the technique by Heath [1-2] and Pool [3] who reported its efficacy in the treatment of patients with cancer and psychiatric conditions. With the advancement of deep brain stimulation in the 1970s, several groups improved the technique by stimulating known targets in the thalamus and internal capsule [4-9]. Further refinement of targets resulted in the stimulation of the PAG/periventricular gray matter (PVG) [10-13] with reproducible and acceptable results. Targeting of the PAG/PVG, with its limited side-effect profile and beneficial patient outcome in some patients, resulted in its continued targeting throughout the next two decades for the treatment of chronic refractory pain $[4,7,12,14]$.

The resurge of interest in the 1990s for the treatment of movement disorders using electrical stimulation resulted in two open-labeled multicenter studies for the use of DBS for pain treatment [15]. These studies failed to show great efficacy of the DBS technology for pain and resulted in the company's abandonment of FDA approval for this modality. Since these studies, several groups have reported small series using DBS for chronic pain [16-19] with benefits suggesting that better patient selection and use of other targets may result in improved outcomes relative to those observed in the trials. Still, other factors may influence patient response to DBS for pain. From the personal experience of the senior author (G.H.), loading patients with 4 grams of L-tryptophan two weeks prior to surgery, and its continual use after surgery, correlates with an excellent response to PAG stimulation for pain. As it relates to deep brain stimulation, Hosobuchi was one of the first to use a similar dose of tryptophan in his patients to prevent the possibility that a limited response to morphine in his infusion trials were the result of tolerance to the analgesic effect of morphine [20]. Indeed, given that tryptophan is the precursor for the neurotransmitter serotonin, others have attempted its use as a stand-alone therapy in the past [20-22]. While the role of serotonin in pain pathways is well established, its concomitant use with DBS targeting of PAG is not well-reported nor understood.

Herein, we report our experience with four patients (Table 1) who were implanted with DBS electrodes at the PAG for nociceptive pain. All patients were loaded with oral L-tryptophan two weeks preoperatively using a dose escalation method consisting of a starting dose of 1 gram and increases of 1 gram every five days as tolerated. The dose of tryptophan was chosen based on doses used by Hosobuchi in the early 1970s [20] and on minimizing the unwanted sideeffects of tryptophan, such as flushing, diarrhea and somnolence. We did not attempt to measure serum serotonin concentrations, nor did we use other formulations of tryptophan in our patients. All PAG electrodes were stereotactically implanted at the same location, 2-3 mm lateral from the wall of the aqueduct and 2-3 mm inferior to the posterior commissure, in each patient using fused MRI CT imaging. Positive contrast ventriculography was used for intraoperative navigation to assess the accuracy of lead placement (+/-500 micrometers). In some patients, a VcPc electrode was also placed in the appropriate somatotopic region of VcPC for a pain syndrome with associated stimulation-induced paraesthesias. Procedures were done under local MAC anesthesia. The PAG target was implanted unilaterally and usually on the contralateral side with typical stimulation parameters of 20-70 ms pulse, with a distal cathode and a proximal cathode combination at 30-40 hertz. No adverse events were associated with intraoperative stimulation and all electrodes were left in situ. Postoperatively, patients were instructed to decrease the tryptophan until stimulation-induced analgesia failed and their pain returned. 


\section{Cureus}

\begin{tabular}{|l|l|l|l|l|l|}
\hline Age & Sex & Pain & Failed Trials & $\begin{array}{l}\text { DBS } \\
\text { Target }\end{array}$ & Improvement \\
\hline 53 & M & Throat & topiramate and lamotrigine & PAG & $\begin{array}{l}70 \% \text { reduction in } \\
\text { methadone dose }\end{array}$ \\
29 & M & $\begin{array}{l}\text { CRPS } \\
\text { II }\end{array}$ & $\begin{array}{l}\text { duloxetine, pregabalin, gabapentin, lidocaine patches, anti- } \\
\text { depressants, ES sympathetic nerve block, spinal cord stimulator }\end{array}$ & $\begin{array}{l}\text { PAG, } \\
\text { VcPc }\end{array}$ & $\begin{array}{l}50 \% \text { reduction in } \\
\text { pain }\end{array}$ \\
\hline 46 & M & Facial & $\begin{array}{l}\text { lidocaine infusions, multiple glycerol rhizotomies, motor cortex } \\
\text { stimulator }\end{array}$ & PAG & $\begin{array}{l}\text { near complete } \\
\text { reduction in pain }\end{array}$ \\
57 & M & Facial & rhizotomy & PAG & $\begin{array}{l}70 \% \text { reduction in } \\
\text { pain }\end{array}$ \\
\hline
\end{tabular}

\section{TABLE 1:}

Summary of the four patients presented in this report, including their age, sex, pain syndrome, failed medical and surgical trials, DBS target implanted and a measure of post-operative improvement.

\section{Case Presentation}

\section{Case 1}

The patient is a 53-year-old man with a long-term history of throat pain secondary to radiation treatment for squamous cell carcinoma of pharynx. The patient reports that he began experiencing pain symptoms immediately following external beam radiation and that his pain was exacerbated by brachytherapy. The pain centered over the right mesial fossa of the sternocleidomastoid muscle. The pain was described as sharp and stabbing, with a burning quality that radiates from the upper neck through the mandible, to the anterior and posterior pinna, and up to the $\mathrm{C} 1$ distribution of the right scalp. He reported exacerbations caused by swallowing, movement and talking. The patient had tried and failed topiramate, phenytoin and lamictal trials. He was subsequently switched to carbamazepine and methadone with moderate pain control but significant cognitive disturbances. Prior to surgical evaluation, the patient's analgesic medications included: methadone $30 \mathrm{mg}$, P.O. in the morning, $20 \mathrm{mg}$, P.O. at noon, and $30 \mathrm{mg}$, P.O. in the evening; carbamazepine X.R. $400 \mathrm{mg}$, p.o. twice daily; venlafaxine S.R. $75 \mathrm{mg}$, S.A. twice daily; nortriptyline, and hydrocodone as needed.

On physical exam, the patient had hyperpathia above his pinna in the C1-C2 distribution, making it impossible to touch his hair. All forms of sensory stimulation, inclusive of light touch, temperature and vibration, produced uncomforting and dysesthetic feeling from vertex down to the sternocleidomastoid. The exam of the posterior of the neck was unremarkable. V1, V2 and V3 were normal, with the exception of base of mandible where he had dysesthetic sensations.

The patient was diagnosed as having radiation neuritis of the ansa cervicalis secondary, and patient was referred to our center for a trial of PAG and VcPc DBS using a Medtronic 3389 and 3387 electrodes, respectively. Postoperative stimulation parameters for the PAG was 0 negative, 1 positive, rate 20/sec, pulse width 120 microsec, amplitude 3.0V and VcPc was 0 negative, 1 positive, rate 180 , pulse width 120 , amplitude $3.0 \mathrm{~V}$. On postoperative day one, the patient complained of frontal headache pain, but was otherwise pain-free. On the evening of postoperative day one, the VcPc stimulator was turned off due to lack of benefit for the patient 
from the stimulation paraesthesia. His PAG electrode was increased to 4.5 volts. On postoperative day two, the patient reported no facial pain with the PAG stimulator and on postoperative day seven, an IPG was implanted and the VcPc DBS electrode explanted. While on 4 grams of oral L-tryptophan daily, the patient experienced a $70 \%$ reduction in his methadone dose. Upon decreasing of the dose to below 2.5 grams L-tryptophan, the patient reported return of previous pain symptoms prior to the PAG DBS implant and restoration of his stimulation analgesia with an increase of his tryptophan dose to 3.5 grams. During the period of failure, attempts were made to re-program the DBS with no success in pain relief. The length of followup for this patient is 10 months and ongoing.

\section{Case 2}

The patient is a 29-year-old male who suffered a left posterior cruciate ligament (PCL) tear in 2006 and subsequent intraoperative accidental severing of the left peroneal nerve, resulting in acute and profound foot drop with severe burning pain in the peroneal nerve distribution on the dorsum of the left foot radiating up into the ankle. The patient had tried several trials of medical management, including duloxetine, pregabalin, gabapentin, lidocaine patches, antidepressants, and one electrosurgical (ES) sympathetic nerve block with minimal benefit. A trial of a spinal cord stimulator resulted in incomplete coverage of the pain symptoms and loss of stimulation efficacy over time.

The patient's preoperative analgesic medications include: hydromorphone $2 \mathrm{mg}$, P.O., every 4 hours; oxycodone / acetaminophen 5/325 mg, P.O., every four hours.

The patient was diagnosed as having Complex Regional Pain Syndrome Type II (CRPS II) secondary to peroneal nerve damage. After stereotactic implantation of the VcPc and PAG sites with Medtronic 3387 and 3389 electrodes, respectively, the PAG-DBS was activated at 0 negative, 1 positive, rate 30, pulse width 120, amplitude 4.0 and VcPc site stimulated at 0 positive, 1 negative, 2 negative, rate 70, pulse width 250, amplitude 2.8. On postoperative day one, the patient reported being pain-free. Although he derived some pain relief from the $\mathrm{VcPc}$, he also noted that the PAG stimulation produced added benefit and insisted on both being internalized. Postoperative day six, the patient had his system internalized with separate IPGs to allow for the different stimulation frequencies of the two sites as listed above. Again, while on 4 grams of oral L-tryptophan daily, the patient experienced a $50 \%$ reduction in pain. Upon decreasing of the dose to below 2.5 grams L-tryptophan, the patient reported return of previous pain symptoms with the DBS functioning. During the period of failure, attempts were made to re-program the DBS with no success in pain relief. The length of follow-up for this patient is three months.

\section{Case 3}

The patient is a 46-year-old man with facial pain, post-resection of a right parotid gland mixed tumor. Three months after his surgery, the patient began to experience right facial pain starting at the edge of his eye and radiating down to his chin. The pain remained refractory to a second resection done for scar tissue and neuroma removal. The patient subsequently tried and failed stereotactic radiosurgical ablation. He had tried multiple trials of medical management, including lidocaine infusions and multiple glycerol rhizotomies. Following minimal benefit of medical treatment, the patient underwent a motor cortex stimulator implantation at our center. While on the motor stimulator (at voltages of 4-4.5), the patient experienced Jacksonian seizures. The episode of seizures were followed by discontinuation of the stimulator, initiation of medical therapy for his newly diagnosed seizure disorder, and the restarting of the stimulator. Unfortunately, at voltages above 3.0, the patient had repeat Jacksonian seizures. The device was interrogated, and amplitude reduced to $0.05 \mathrm{~V}$ and left on (0-3 negative, 4-7 positive, rate 75 , pulse width 450 , amplitude 0.05 ). 
The patient's analgesic medications include: zolpidem CR $12.5 \mathrm{mg}$, P.O., at bed time; Carbamazepine $900 \mathrm{mg}$, P.O. every morning, and $1200 \mathrm{mg}$, P.O. at dinner; lamotrigine $100 \mathrm{mg}$, P.O., every morning and $200 \mathrm{mg}$, P.O. at dinner; and ativan $2 \mathrm{mg}$, P.O., with dinner.

The patient was diagnosed as having trigeminal neuropathy secondary to the resection of the parotid tumor and irradiation. Given the patient's complicated treatment history, and due to the non-opiate responsiveness of his pain, he elected to undergo PAG stimulation. A 3389 DBS electrodes were placed at the PAG at 0 negative, 1 positive, rate 40 , pulse width 120 , amplitude $6.5 \mathrm{~V}$. On postoperative day one, the patient reported being pain-free. Once again, while on 4 grams of L-tryptophan, the patient experienced a near complete reduction in pain. Upon decreasing of the dose to below 2.5 grams L-tryptophan, the patient reported return of previous pain symptoms with the DBS in place. During the period of failure, attempts were made to reprogram the DBS with no success in pain relief. The length of follow-up for this patient is 14 months.

\section{Case 4}

The patient is a 57-year-old man with left facial pain secondary to sinus surgery in 1987. The patient describes the pain as numbness and burning over his left V1 as well as V1 dermatomes. Following the failure of medical treatment, the patient underwent rhizotomies with onset of left anesthesia dolorosa. After this procedure, the patient had implantation of a Gasserian stimulator and an implantable pulse generator (1995) with some benefit with the pain. Shortly after the insertion of the Gasserian stimulator, the stimulator became infected, necessitating its removal, and he was subsequently implanted with a right motor cortex stimulator by the senior author in 2005. He escaped motor cortex stimulation in 2007 and went on to a DBS trial. The patient's past medical history is significant for seasonal allergies, benign essential tremor of upper extremities, chronic left facial pain, anesthesia dolorosa, and hyperlipidemia.

The patient's medications include: clonidine $0.2 \mathrm{mg}$, P.O., four times a day; baclofen $20 \mathrm{mg}$, P.O., three times a day; gabapentin $600 \mathrm{mg}$, P.O., four times a day; levetiracetam $750 \mathrm{mg}$, P.O., daily; klonopin $1 \mathrm{mg}$, P.O., twice a day; Levo-Dromoran $4 \mathrm{mg}$, P.O., four times a day; and tryptophan $4 \mathrm{mg}$ daily.

The patient was diagnosed as having trigeminal neuropathy secondary to his sinus surgery. DBS electrodes were placed at the PAG at 0 negative, 1 positive, rate 25 , pulse width 100 , amplitude 1.6. On postoperative day one, the patient reported being pain-free. Once again, while on 4 grams of oral L-tryptophan daily, the patient experienced a $70 \%$ reduction in his pain symptoms. Postoperatively, the patient complained of somnolence while taking 4 grams of oral L-tryptophan daily. As a result, the dose was reduced to 3 grams with immediate return of the pain symptoms. Subsequently, the patient was switched to $4 \mathrm{mg}$ of oral melatonin daily with resolution of the pain. He is not using his motor cortex stimulator at this time. During the period of failure, attempts were made to re-program the DBS with no success in pain relief. The length of follow-up for this patient is 12 months.

\section{Discussion}

The PAG has been a target of DBS for intractable nociceptive pain for longer than three decades. Although the exact mechanism of DBS and the neural circuits modulating pain at the PAG are ill-defined, peripheral evidence suggest that pain alleviation is caused by local release of endogenous endorphins via a serotonin-mediated pathway [23].

\section{The circuitry of pain}

There are three important pathways for the relay of painful stimuli in the spinal cord: 1) the 
spinothalamic tract which is the prominent pathway for the relay of pain in the spinal cord. Stimuli arrive at the spinal cord via peripheral nerves and synapse in the dorsal horn, decussate to the contralateral side, and travel rostrally [21]. These fibers terminate at the ventroposterolateral nucleus (VPL) where they connect with the somatosensory cortex; 2) the spinoreticular tract which is responsible for the motivational response to pain. Nociceptive or slow pain fibers belonging to this tract terminate at the level of the reticular formation in the pons and the medulla and then project to the intralamina of the thalamus, the hypothalamus, the cingulate gyrus, and the limbic system [21]; and 3) the spinomesencephalic tract which is responsible for affective pain, contains fibers that ascend to terminate in the superior colliculus, PAG, and nucleus cuneiformis [21].

\section{Periaqueductal gray (PAG) and pain}

The role of the PAG in the spinomesencephalic pathway of pain is not well-understood. Electrical stimulation of the PAG produces analgesia in several animal models [24-26] and patients [10]. Experimental studies are inconclusive as to the exact pathways of analgesia. Studies in rodents suggest that the PAG activates an inhibitory cascade via descending serotonergic and noradrenergic pathways in the rostroventral medulla and pontine noradrenergic nuclei, causing an ablation of the sensation of nociceptive pain [27]. Other groups have suggested that an excitatory connection between the periaqueductal gray and the nucleus raphe magnus (NRM) and that activation of this system can cause analgesia [28]. Still others have shown that morphine administered to the periaqueductal gray is capable of activating an ascending serotonergic pathway to release S-hydroxytryptamine in the nucleus accumbens, which in turn activates an enkephalinergic mechanism within the same nucleus, resulting in an anti-nociceptive effect [29].

What is common to all these studies is that signaling pathways involved in both opiate and electrically-induced analgesia are serotonin-mediated. Peripherally, there is correlative, necessary and sufficient evidence linking serotonin precursors and by-products in the PAG pain circuitry: 1) Paraventricular thalamic nucleus of cynomolgus monkeys contain a high density of axons immunoreactive for serotonin [30]. The anti-nociceptive effects resulting from electrical stimulation the periaqueductal gray can be blocked by the administration of serotonin receptor blockers in primates [22]. And in the case of humans, as our four cases illustrate, the addition of serotonin to the pre- and postoperative regimen of the patient results in enhanced alleviation of pain. We hypothesize, with much experimental evidence in support of our hypothesis, that the loading of patients with tryptophan provides the body with an excess of the starting material used for serotonin synthesis. The abundance of serotonin can activate the aforementioned serotonin-dependent pathways and provide analgesia. Although we cannot provide direct evidence that the L-tryptophan loading is directly influencing one of the aforementioned pathways, there is strong evidence from other sources that we are modulating the serotonergic circuit of pain using our loading regimen.

\section{Conclusions}

Although DBS of PAG for pain has had a mixed history of success and failure, recent evidence from small series suggest that it may be a viable option for select patients. Herein, we present four cases of patients implanted at our institution with electrodes at the PAG for intractable nociceptive pain. What is unique about our procedure, and we believe the key to the success in our patients, is the pre- and postoperative loading of tryptophan. The increased serum concentration of tryptophan, very likely, causes an increase in serum serotonin concentrations and activation of one of the anti-nociceptive pathways described.

\section{Additional Information}




\section{Disclosures}

Human subjects: Consent was obtained by all participants in this study. Conflicts of interest: In compliance with the ICMJE uniform disclosure form, all authors declare the following:

Payment/services info: All authors have declared that no financial support was received from any organization for the submitted work. Financial relationships: All authors have declared that they have no financial relationships at present or within the previous three years with any organizations that might have an interest in the submitted work. Other relationships: All authors have declared that there are no other relationships or activities that could appear to have influenced the submitted work.

\section{References}

1. Heath R: Studies in schizophrenia: a multidisciplinary approach to mind-brain relationships . Harvard University Press, Cambridge; 1954.

2. Heath R, Mickle W: Evaluation of seven years' experience with depth electrode studies in human patients. Electrical studies on the unanesthetized human brain. Ramey E, O'Doherty D (ed): Paul B. Hoeber, Inc., New York; 1960. 214-247.

3. Pool J, Clark W, Hudson P, Lombardo M: Steroid hormondal response to stimulation of electrodes implanted in the subfrontal parts of the brain. Hypothalamic-hypophysial Interrelationships, a Symposium. Fields W, Guillemin R, Carton C (ed): Charles C. Thomas, Springfield; 1956. 114-124.

4. Adams JE, Hosobuchi Y, Fields HL: Stimulation of internal capsule for relief of chronic pain . J Neurosurg. 1974, 41:740-744.

5. Fields HL, Adams JE: Pain after cortical injury relieved by electrical stimulation of the internal capsule. Brain. 1974, 97:169-178.

6. Hosobuchi Y, Adams JE, Rutkin B: Chronic thalamic stimulation for the control of facial anesthesia dolorosa. Arch Neurol. 1973, 29:158-161.

7. Hosobuchi Y, Adams JE, Rutkin B: Chronic thalamic and internal capsule stimulation for the control of central pain. Surg Neurol . 1975, 4:91-92.

8. Mazars G, Merienne L, Cioloca C: Treatment of certain types of pain with implantable thalamic stimulators. Neurochirurgie. 1974, 20:117-124.

9. White J, Sweet W: Pain and the Neurosurgeon: a 40 year experience . Charles C. Thomas, Springfield; 1969.

10. Hosobuchi Y, Adams JE, Linchitz R: Pain relief by electrical stimulation of the central gray matter in humans and its reversal by naloxone. Science. 1977, 197:183-186.

11. Richardson DE, Akil H: Long term results of periventricular gray selfstimulation. Neurosurgery. 1977, 1:199-202.

12. Richardson DE, Akil H: Pain reduction by electrical brain stimulation in man. Part 1: Acute administration in periaqueductal and periventricular sites. J Neurosurg. 1977, 47:178-183.

13. Richardson DE, Akil H: Pain reduction by electrical brain stimulation in man. Part 2: Chronic self-administration in the periventricular gray matter. J Neurosurg. 1977, 47:184-194.

14. Mazars GJ: Intermittent stimulation of nucleus ventralis posterolateralis for intractable pain . Surg Neurol. 1975, 4:93-95.

15. Coffey RJ: Deep brain stimulation for chronic pain: results of two multicenter trials and a structured review. Pain Med. 2001, 2:183-192.

16. Hamani C, Schwalb JM, Rezai AR, Dostrovsky JO, Davis KD, Lozano AM: Deep brain stimulation for chronic neuropathic pain: long-term outcome and the incidence of insertional effect. Pain. 2006, 125:188-196.

17. Kumar K, Toth C, Nath RK: Deep brain stimulation for intractable pain: a 15-year experience . Neurosurgery . 1997, 40:736-746.

18. Nandi D, Aziz TZ: Deep brain stimulation in the management of neuropathic pain and multiple sclerosis tremor. J Clin Neurophysiol . 2004, 21:31-39.

19. Tasker RR, Vilela Filho O: Deep brain stimulation for neuropathic pain. Stereotact Funct Neurosurg. 1995, 65:122-124.

20. Hosobuchi Y: Combined electrical stimulation of the periaqueductal gray matter and sensory thalamus. Appl Neurophysiol. 1983, 46:112-115. 
21. Mumenthaler M, Mattle H, Taub E: Fundamentals of Neurology. Thieme, New York; 2006.

22. Yezierski RP, Wilcox TK, Willis WD: The effects of serotonin antagonists on the inhibition of primate spinothalamic tract cells produced by stimulation in nucleus raphe magnus or periaqueductal gray. J Pharmacol Exp Ther. 1982, 220:266-277.

23. Sillery E, Bittar RG, Robson MD, Behrens TE, Stein J, Aziz TZ, Johansen-Berg H: Connectivity of the human periventricular-periaqueductal gray region. J Neurosurg. 2005, 103:1030-1034.

24. Lewis VA, Gebhart GF: Evaluation of the periaqueductal central gray (PAG) as a morphinespecific locus of action and examination of morphine-induced and stimulation-produced analgesia at coincident PAG loci. Brain Res. 1977, 124:283-303.

25. Mayer DJ, Wolfle TL, Akil H, Carder B, Liebeskind JC: Analgesia from electrical stimulation in the brainstem of the rat. Science. 1971, 174:1351-1354.

26. Oliveras JL, Besson JM, Guilbaud G, Liebeskind JC: Behavioral and electrophysiological evidence of pain inhibition from midbrain stimulation in the cat. Exp Brain Res . 1974, 20:3244.

27. Basbaum AI, Clanton CH, Fields HL: Opiate and stimulus-produced analgesia: functional anatomy of a medullospinal pathway. Proc Natl Acad Sci U S A . 1976, 73:4685-4688.

28. Behbehani MM, Fields HL: Evidence that an excitatory connection between the periaqueductal gray and nucleus raphe magnus mediates stimulation produced analgesia. Brain Res. 1979, 170:85-93.

29. Xuan YT, Shi YS, Zhou ZF, Han JS: Studies on the mesolimbic loop of antinociception--II. A serotonin-enkephalin interaction in the nucleus accumbens. Neuroscience. 1986, 19:403-409.

30. Hsu DT, Price JL: Paraventricular thalamic nucleus: subcortical connections and innervation by serotonin, orexin, and corticotropin-releasing hormone in macaque monkeys. J Comp Neurol. 2009, 512:825-848. 